\title{
Comparison of Kaposi disease outlines in patients with and without HIV infection in two tertiary care hospitals in Bucharest, Romania
}

\author{
Oana Săndulescu ${ }^{1,2^{*}}$, loana Țiu ${ }^{3}$, Raluca Jipa ${ }^{2}$, Anca Streinu-Cercel ${ }^{1,2}$, Șerban Benea ${ }^{1,2}$, Olga Simionescu ${ }^{1,3}$, \\ Adrian Streinu-Cercel ${ }^{1,2}$, Adriana Hristea ${ }^{1,2}$ \\ From The 10th Edition of the Scientific Days of the National Institute for Infectious Diseases "Prof Dr Matei \\ Bals" \\ Bucharest, Romania. 15-17 October 2014
}

\section{Background}

Kaposi disease (KD) displays polymorphic manifestations, ranging from minimal cutaneous involvement to extensive visceral disease. Its clinical outline is different in HIV-positive patients compared to the classical nonHIV-related form that is diagnosed more often in elderly patients without other significant immune impairment.

\section{Methods}

A retrospective study on KD was performed in two academic centers, tertiary-care hospitals with national addressability in Romania. Two groups were comparatively studied: HIV-infected patients diagnosed in the National Institute for Infectious Diseases "Prof.Dr. Matei Balş" (HIV-positive group), and non-HIV patients diagnosed in the first Clinic of Dermatology, Colentina Clinical Hospital (HIV-negative group). The statistical analysis was performed using IBM SPSS Statistics v.22 (Chicago, USA).

\section{Results}

A total number of 71 cases, 30 in the HIV-positive group and 41 in the HIV-negative group were identified. The non-HIV patients were benign European form and immunosuppressed other than HIV, respectively. There was a male predominance, with a male-to-female ratio in HIV-positive and HIV-negative patients of 2:1 and 4.1:1 respectively. The mean age at KD diagnosis was

\footnotetext{
* Correspondence: oanasandulescu1@gmail.com

${ }^{1}$ Carol Davila University of Medicine and Pharmacy, Bucharest, Romania

Full list of author information is available at the end of the article
}

41.6 \pm 15.0 years in HIV-positive and $70.2 \pm 11.8$ years in HIV-negative patients.

The mean number of comorbidities was 3 in the HIVpositive group compared to 1 in HIV-negative, $\mathrm{p}=$ 0.011). In the HIV-positive group, 17 patients (56.7\%) were classified Mitsuyasu stage 1, 6 (20\%) stage 2, 2 (6.7\%) stage 3 and 5 (16.7\%) stage 4. In the HIV-negative group, all 41 patients were Mitsuyasu stage 1.

In the HIV-positive group all patients received antiretroviral therapy and only $6(20 \%)$ received other types of targeted therapy for KD: topical $(5,16.7 \%)$, systemic (5, $16.7 \%$ - interferon, etc.), chemotherapy (2, 6.7\%), local radiotherapy $(2,6.7 \%)$. In the HIV-negative group, all patients received specific treatment, such as: topical (24, $58.5 \%)$, systemic $(14,34.1 \%$ - dapsone, pentoxifylline), chemotherapy $(2,4.9 \%)$, local radiotherapy $(7,17.1 \%)$, electrocauterization $(15,36.6 \%)$, surgical excision $(4,9.8 \%)$.

Loss for follow-up appeared to be less frequent in the HIV-positive group (7 patients, 35\%) than in the HIVnegative group (35 patients, $85.4 \%$ ) but occurred faster in the HIV-positive group, after a mean interval of $8.6 \pm$ 3.4 vs. $15.9 \pm 37.3$ months in HIV-negative group. Overall survival was 60\% (HIV-positive) and 100\% (HIVnegative).

\section{Conclusion}

This work has identified two different outlines of KD, an aggressive progression with high mortality in HIV-positive patients, and a gradual progression, with virtually no short-term mortality, in elderly patients. In the HIVpositive group, very few patients received other types of treatment for $\mathrm{KD}$, apart from antiretroviral therapy. 


\section{Authors' details}

${ }^{1}$ Carol Davila University of Medicine and Pharmacy, Bucharest, Romania.

${ }^{2}$ National Institute for Infectious Diseases "Prof. Dr. Matei Balş", Bucharest, Romania. ${ }^{3}$ First Department of Dermatology, Colentina Clinical Hospital,

Bucharest, Romania.

Published: 15 October 2014

doi:10.1186/1471-2334-14-S7-P20

Cite this article as: Săndulescu et al:: Comparison of Kaposi disease outlines in patients with and without HIV infection in two tertiary care hospitals in Bucharest, Romania. BMC Infectious Diseases 2014 14(Suppl 7): P2O.

Submit your next manuscript to BioMed Central and take full advantage of:

- Convenient online submission

- Thorough peer review

- No space constraints or color figure charges

- Immediate publication on acceptance

- Inclusion in PubMed, CAS, Scopus and Google Scholar

- Research which is freely available for redistribution

Submit your manuscript at www.biomedcentral.com/submit 